\title{
BMJ Open Acupuncture for chronic cancer-related pain: a systematic review and network meta-analysis protocol
}

\author{
Jiao Yang (1) , Guixing Xu (D) , Zihan Yin (D), Ying Cheng (D), Sheng Ming Sun, \\ Qianhua Zheng, Jiao Chen (D) , Fan-rong Liang (D) , Ling Zhao
}

To cite: Yang J, Xu G, Yin Z, et al. Acupuncture for chronic cancer-related pain: a systematic review and network meta-analysis protocol. BMJ Open 2020;10:e039087. doi:10.1136/ bmjopen-2020-039087

- Prepublication history and additional material for this paper is available online. To view these files, please visit the journal online (http://dx.doi.org/10. 1136/bmjopen-2020-039087).

Received 03 April 2020 Revised 03 June 2020 Accepted 03 August 2020
Check for updates

(c) Author(s) (or their employer(s)) 2020. Re-use permitted under CC BY-NC. No commercial re-use. See rights and permissions. Published by BMJ.

Acupuncture and Tuina School, Chengdu University of Traditional Chinese Medicine, Chengdu, Sichuan, China

Correspondence to Dr Fan-rong Liang; acuresearch@126.com and Professor Ling Zhao; zhaoling@cdutcm.edu.cn

\section{ABSTRACT}

Introduction Chronic cancer-related pain is one of the most common excruciating symptom that can be caused by the cancer (by the primary tumour or by metastases) or by its treatment (surgery, chemotherapy and radiotherapy). Although multiple clinical trials and systematic reviews have suggested that acupuncture could be effective in treating chronic cancer-related pain, the comparative efficacy and safety of these acupuncture methods remains unclear. We, therefore, performed this study to evaluate and rank the efficacy and safety of different acupuncture methods for chronic cancer-related pain.

Methods and analysis Seven databases will be searched, including Cochrane Library, MEDLINE, Embase, Chinese National Knowledge Infrastructure (CNKI), Wanfang Database, the Chongqing VIP Chinese Science and Technology Periodical Database and Chinese Biomedical Literature Database (CBM) from their inception to March 2020. The primary outcome is the change of pain intensity. Bayesian network meta-analysis will be conducted using software R3.5.1. Finally, we will use the Grading of Recommendations Assessment, Development and Evaluation System (GRADE) to assess the quality of evidence.

Ethics and dissemination Ethical approval is not required for literature-based studies. The results will be disseminated through peer-reviewed publication. PROSPERO registration number CRD42020165747

\section{INTRODUCTION}

Chronic cancer-related pain is one of the most common symptoms in cancer patients, ${ }^{1}$ which includes chronic cancer pain and chronic post-cancer treatment pain. ${ }^{2}$ Studies showed that the incidence of chronic cancer-related pain is established to be $33 \%$ for patients after curative treatment, $59 \%$ for patients undergoing anticancer treatment and $64 \%$ for patients with metastatic, advanced or terminal disease ${ }^{34}$ Particularly, pain is highly prevalent in early stages in certain cancer types such as pancreatic cancer $(44 \%)$ and head and neck cancer $(40 \%) .{ }^{5}$ It could lead to mood disturbance, dyspepsia and poor quality of life. ${ }^{67}$ In terms of treatment approach, the WHO analgesic ladder recommends opioid therapy

\section{Strengths and limitations of this study}

- This study will be the first of its kind to compare the efficacy and safety of various acupuncture methods in the treatment of chronic cancer-related pain using Bayesian network meta-analysis.

- The quality of evidence will be assessed by the Grading of Recommendations Assessment, Development and Evaluation system (GRADE).

- The study will be carried out according to the recommendation of Cochrane handbook for systematic reviews of interventions.

- We will only retrieve data from Chinese and English databases, which could limit available data or result in language bias.

- The quality of the pooled effects will be affected by original trials.

on the basis of pain intensity. ${ }^{8}$ However, over half of all cancer patients still suffer intolerable pain. ${ }^{4}$ The inadequate management of chronic cancer-related pain have a significant harmful impact on the quality of life for patients ${ }^{9}$ and may lead to increased healthcare costs. ${ }^{10}$ Moreover, many patients develop adverse effects from analgesic regimen, such as constipation, nausea, drowsiness, confusion and hallucinations. ${ }^{11} 12$ Each adverse effect requires a careful assessment and treatment strategy, and increase the financial burden of patients. ${ }^{13}$ Therefore, it is necessary to explore other forms of alternative therapies which are both safe and effective in relieving chronic cancer-related pain.

The USA and Europe have developed guidelines on complementary and alternative medicine (CAM) for chronic cancerrelated pain; ${ }^{14}$ most patients use CAM as an adjunct therapy along with the conventional treatments. ${ }^{15}$ As one of CAM treatments, acupuncture plays an important role in the treatment of pain. ${ }^{16-18}$ In recent years, various acupuncture methods have been widely used in treating chronic cancerrelated pain and adverse effects related 
to the cancer treatments. ${ }^{19-24}$ Most National Cancer Institute-designated comprehensive cancer centres have begun offering acupuncture. In addition, systematic reviews showed that acupuncture and/or acupressure is significantly associated with reducing chronic cancerrelated pain and decreasing the use of analgesics. ${ }^{20} 21$ Another Cochrane systematic review showed that all studies reported benefits of acupuncture in managing pancreatic cancer pain. ${ }^{22}$ The comparison between acupuncture plus drug therapy, and drug therapy alone demonstrated a significant favouring of acupuncture plus drug therapy. ${ }^{23} \mathrm{~A}$ randomised control trial indicates the efficacy of auricular acupuncture for patients receiving chemotherapy. ${ }^{24}$ However, due to the diversity of acupuncture approach, its relative effectiveness has not yet been studied or explained. Clinicians are confused about how to choose the optimal acupuncture method for chronic cancer-related pain.

Studies showed that the rankings of different treatments can be provided using the network meta-analysis (NMA) to analyse the direct and indirect randomised data. 2526 Therefore, we will perform this NMA to comprehensively evaluate the effectiveness of various acupuncture therapies for chronic cancer-related pain.

\section{Objective}

The purpose of this study is to compare the efficacy and safety of existing acupuncture methods for the treatment of chronic cancer-related pain through NMA and systematic review.

\section{METHODS}

This protocol will be conducted according to the Preferred Reporting Items for Systematic Reviews and Meta-Analyses Protocols (PRISMA-P) statement and the Checklist of Items to Include When Reporting a Systematic Review Involving a Network Meta-analysis. ${ }^{27} 28$ The research has been registered on PROSPERO (online supplemental file 1 for PRISMA-P checklist).

\section{Criteria for including studies in this review \\ Types of studies}

The review will include randomised controlled trials (RCTs) that were reported in English or Chinese without any regional restrictions. The first period of randomised cross-over trials will be included. Non-RCT reviews, case report, animal experimental studies, expert experience, conference article and duplicated publications will be excluded.

\section{Types of participants}

We will include patients with chronic cancer-related pain, which includes chronic cancer pain and chronic post-cancer treatment pain, regardless of the cancer type.

We will define chronic cancer-related pain as pain directly linked to the development of cancer confirmed by pathology or radiology. Trials that studied chronic cancer-related pain mixed with other types of pain and trials that studied chronic post-cancer surgery pain will be excluded.

\section{Types of interventions}

We will define acupuncture in this review as acupointbased therapy, regardless of needling techniques and stimulation method, including manual acupuncture, electro-acupuncture, auricular (ear) acupuncture, acupressure, acupoint application, moxibustion, catgut embedding, transcutaneous electrical acupoint stimulation, acupoint injection and others. We will rule out interventions without stimulating the acupoint.

\section{Types of control groups}

Treatments in the comparison groups can be shamacupuncture, placebo, pharmacotherapy or no additional intervention to usual care. Studies that compared different types of acupoint-based therapy will be included.

\section{Types of outcome measures}

Studies reporting one or more of the following outcomes will be included.

\section{Primary outcomes}

The change of pain intensity will be measured by a visual analogue scale (VAS), ${ }^{29}{ }^{30}$ McGill Pain Questionnaire (MPQ), ${ }^{31}{ }^{32}$ Brief Pain Inventory (BPI) ${ }^{33}$ or other validated outcome measures.

\section{Secondary outcomes}

1. Quality of life measured by validated scales including the European Organisation for Research and Treatment of Cancer Quality of Life Questionnaire (EORTC-QLQC30), the General Version of the Functional Assessment of Cancer Therapy (FACT-G), the Edmonton Symptom Assessment System (ESAS) or other validated scales. ${ }^{34}$

2. Consumption of analgesics, including opioids and non-opioids. ${ }^{35}$

3. Frequency of breakthrough pain and rescue medication use or dosage.

4. Side effects of analgesic regimen, such as nausea and vomiting, constipation and cognitive deficits.

5. Safety of the acupoint-based therapies, including adverse events and withdrawals for any reason.

\section{Search methods for identification of studies}

The following databases will be searched from their inception to March 2020: Cochrane Library, MEDLINE, Embase, Chinese National Knowledge Infrastructure (CNKI), Wanfang Database, the Chongqing VIP Chinese Science and Technology Periodical Database, Chinese Biomedical Literature Database (CBM), WHO Clinical Trials Registry, Chinese clinical registry, ClinicalTrials. gov and reference lists of articles to identify additional studies. 


\begin{tabular}{|c|c|}
\hline Number & Search items \\
\hline 1 & exp acupuncture therapy \\
\hline 2 & exp medicine, east asian traditional \\
\hline 3 & exp acupuncture \\
\hline 4 & $\begin{array}{l}\text { (acupuncture or acupoint* or electroacupuncture } \\
\text { or electro-acupuncture or meridian* or } \\
\text { moxibustion* or "traditional Chinese } \\
\text { medicine" or "traditional oriental medicine" or } \\
\text { auriculotherapy or needle or "acupoint catgut } \\
\text { embedding" or "wrist-ankle acupuncture" } \\
\text { or "scalp acupuncture" or "transcutaneous } \\
\text { electrical acupoint stimulation" or "acupoint } \\
\text { injection").mp }\end{array}$ \\
\hline 5 & Or 1 to 4 \\
\hline 6 & exp neoplasms \\
\hline 7 & $\begin{array}{l}\text { (neoplasm* or cancer }{ }^{\star} \text { or carcino* or malignan* } \\
\left.\text { or tumor }{ }^{*} \text { or tumour }{ }^{*}\right) . \mathrm{mp}\end{array}$ \\
\hline 8 & Or 6 to 7 \\
\hline 9 & exp pain \\
\hline 10 & pain*.mp. \\
\hline 11 & exp analgesia \\
\hline 12 & (analges* or nocicept* or neuropath $\left.{ }^{\star}\right) . m p$ \\
\hline 13 & Or 9 to 12 \\
\hline 14 & 13 and 8 and 5 \\
\hline 15 & randomised controlled trial.pt \\
\hline 16 & controlled clinical trial.pt \\
\hline 17 & randomised.ab \\
\hline 18 & placebo.ab \\
\hline 19 & drug therapy.fs \\
\hline 20 & randomly.ab \\
\hline 21 & trial.ab \\
\hline 22 & groups.ab \\
\hline 23 & Or 15 to 22 \\
\hline 24 & exp animals/ not humans.sh \\
\hline 25 & 23 not 24 \\
\hline 26 & 25 and 14 \\
\hline
\end{tabular}

This significance of ${ }^{1 * 1}$ means that phrase including the term before ${ }^{1 * 1}$ can be searched.

The following medical search headings (MeSH) will be used: "cancer", " tumor", "carcinoma", "neoplasms", "pain", "analgesia", "acupuncture", "electro acupuncture", "auriculotherapy", "acupoint", "needle", "acupoint catgut embedding", "wrist-ankle acupuncture", "moxibustion", "scalp acupuncture", "transcutaneous electrical acupoint stimulation"," acupoint injection", "randomized controlled trial"," randomised controlled", "randomised, controlled" and "clinical trial". Chinese translations of these search terms will be used for the Chinese databases. The search strategy for MEDLINE is shown in table 1.

\section{Data collection and analysis}

Selection of studies

Two reviewers (JY and GX) will screen all hits independently based on the titles and abstracts. Full texts will be downloaded for further evaluation when necessary. By the next stage, the reviewers will examine the full-text articles according to the inclusion criteria. A third reviewer (QZ) will be consulted to resolve any disagreement by discussion and consensus. The selection procedure will be shown in a PRISMA flow chart (figure 1)

\section{Data extraction and management}

Two independent reviewers(ZHYn and MSS) will extract information using a pre-designed form including: (1) identification information (publication year, first author); (2) general information (country, study type, number of centres, sample size and study design); (3) participants (type and/or stage of cancer, age, sex and pain intensity before treatment); (4) interventions (type of acupuncture, acupuncture points selection and treatment frequency/session/duration); (5) comparator (if there is any, details of the treatment including name, dosage, frequency and course); and (6) outcomes (data and time points for each measurement, and safety).

We will try to contact corresponding authors for missing data or clarification for unclear information. Any disagreements will be arbitrated by a third reviewer (LZ). Cross-check of all data will be done by ZHY and MSS before transfer into RevMan software (V.5.3).

\section{Quality assessment}

Two or more independent reviews (YC and JC) will appraise the quality of the included trials using the risk of bias tool developed by the Cochrane Collaboration. ${ }^{36}$ We will appraise each study in terms of selection bias (random sequence generation and allocation concealment), performance bias (blinding of participants and personnel), detection bias (blinding of outcome assessment), attrition bias (incomplete outcome data), selective reporting bias and other bias. Trials will be evaluated and classified into three levels: low risk, high risk and unclear. Any disagreements will be arbitrated by a third reviewer (LZ).

The Grading of Recommendations Assessment, Development and Evaluation System (GRADE) system will be used to grade the quality of the evidence for main outcomes. ${ }^{37}$ Two reviewers will use the GRADE system to independently assess the quality of evidence for each outcome. Evidence quality will be rated 'high', 'moderate', 'low' or 'very low' according to the GRADE rating standards. The quality of evidence of a specific study will be assessed according to the risk of bias, inconsistency, indirectness, imprecision and publication bias.

\section{Assessment of similarity and consistency}

An assessment of similarity and consistency will be performed to produce a credible and valid result. Since it is difficult to determine similarity using statistical analysis, 

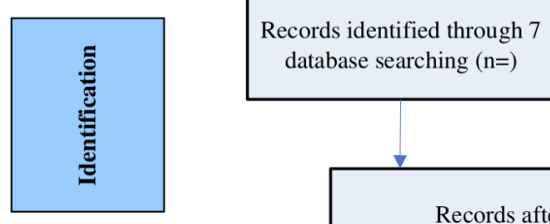

Additional records identified database searching $(\mathrm{n}=)$
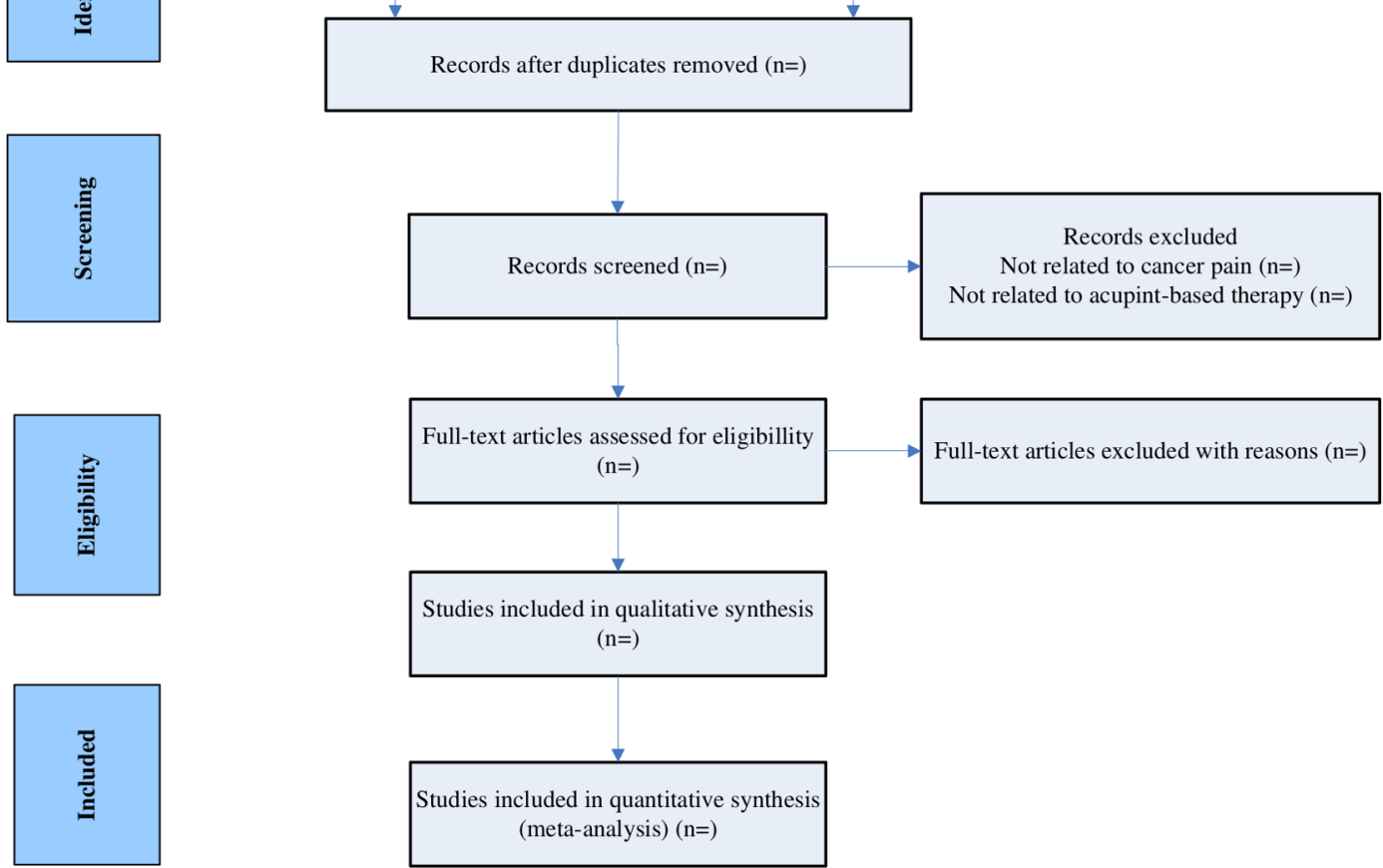

Figure 1 PRISMA flow diagram of the study selection process. PRISMA, Preferred Reporting Items for Systematic Reviews and Meta-Analyses.

the assessment will be based on clinical and methodological characteristics, including study designs, participant characteristics and interventions. We will conduct the z-test to check the consistency, and the $p$ value will be calculated to confirm whether there are inconsistencies among the comparison of direct and indirect. If $p>0.05$, the comparison of direct and indirect is consistent; on the contrary, the comparison will be considered inconsistent.

\section{Network meta-analysis}

Efficacy data will be synthesised and statistically analysed in R3.5.1 with the Bayesian method. ${ }^{38}$ Dichotomous data will be investigated by using a risk ratio with $95 \%$ CIs. For continuous outcomes, data will be analysed by using a standard mean difference with $95 \%$ CIs or a weighted mean difference. The weighted mean difference will be used for the same scale or the same assessment instrument, whereas the standard mean difference will be used for different assessment tools.

The contribution of different designs to the final effect size of the NMA will be evaluated by net-heat plots. The acupoint-based therapies will be ranked by using $p$ score that measures the extent of certainty when treatment is better than control. A p score equals $100 \%$ when a treatment is certain to be the best and $0 \% \mathrm{p}$ score indicates a treatment to be the worst. We will use the forest plots to present the results of NMA. Ranking of the different acupuncture methods will be displayed according to the surface under the cumulative ranking curve analysis
(SUCRA). Network plot will be used to show the comparisons between interventions.

\section{Assessment of heterogeneity}

Clinical and methodological heterogeneity will be evaluated by closely checking the features of the participants, interventions and outcomes of the inclusive studies, and comparing fit of the fixed effect model and random effect model. Statistical heterogeneity will be assessed by the $I^{2}$ index. Values of $I^{2}<50 \%$ will indicate that heterogeneity is not salient for the cases that we explored; otherwise, substantial heterogeneity will be considered. ${ }^{37}$ Meta-analysis will be performed after removal of studies where main or unacceptable sources of heterogeneity were derived. Furthermore, if the source of heterogeneity cannot be explored, a narrative review will be provided.

\section{Meta-regression, subgroup analysis and sensitivity analysis}

A network meta-regression will be performed to explore sources of heterogeneity using a random effects network meta-regression model. If sufficient evidence is available, we will conduct subgroup analyses based on cancer types and degree of pain. In order to obtain a stable conclusion, a sensitivity analysis will be conducted to remove effects of trials with small sample size and remove studies rated as high risk of bias based on accounting of methodological quality. These steps will be crucial to ensure the accuracy and depth of inferences from results. 


\section{Patients and public involvement}

There were no patients nor public will be directly involved in this review. Only data already existent in the literature and the aforementioned sources will be used for this study.

\section{DISCUSSION}

Pain often affects cancer patients and represents a major challenge for both clinicians and patients. ${ }^{39}$ More than one-third of patients with cancer rate their pain as moderate-to-severe in nature. ${ }^{4}$ In most National Cancer Institutions, acupuncture has a decisive role in the treatment of chronic cancer-related pain, but acupuncture therapies for chronic cancer-related pain are diverse. Clinicians are confused to select the optimal one. However, exploring the most suitable acupuncture methods may not only increase financial burden but also waste medical resources.

NMA can be used to integrate direct and indirect comparisons across a set of multiple variables, it can help evaluate the comparative efficacy and safety of various acupuncture methods. ${ }^{40}{ }^{41}$ Bayesian methods involve a formal combination of a prior probability distribution with a (likelihood) distribution of the pooled effect based on the observed data to obtain a posterior probability distribution of the pooled effect. ${ }^{42}$ Compared with frequency methods, Bayesian methods can naturally lead to a decision framework to support decisionmaking. ${ }^{42-44}$ This overcomes the defect of the frequency method in parameter estimation, which estimates the maximum likelihood through continuous iteration and causes unstable results. Moreover, Bayesian meta-analysis is straightforward in making predictions and it is possible to incorporate different sources of uncertainty, ${ }^{42} 44$ which was recommended for NMA. Therefore, efficacy data will be synthesised and analysed with Bayesian method in our review. Based on the type of single study, we will conduct a rigorous analysis of multiple inclusion criteria and quality scores for results evaluated by GRADE. ${ }^{45}$

To the best of our knowledge, this study will be the first systemic review (SR) and NMA to investigate acupuncture therapies for chronic cancer-related pain. Based on evidence of comparative effectiveness and safety, the NMA is expected to provide a ranking of these methods for cancer patients suffering from pain. Moreover, the NMA may assist patients, physicians and clinical research investigators to choose the most appropriate acupuncture method. Finally, we sincerely hope that our results will offer credible evidence for the clinicians and encourage wider application of acupuncture for chronic cancerrelated pain.

\section{Ethics and dissemination}

The results will be disseminated through peer-reviewed journals or conference reports. There are no ethical considerations related to the agreement, since no private data will be included in the SR. We will not endanger the individual's privacy or compromise their rights.

Contributors JY, ZY and LZ conceived this study. JY, GX and ZY will develop the study protocol and will implement the systematic review under the supervision of JC and QZ. GX will provide the statistical analysis plan of the study and will conduct data analysis. YC and MS will perform the study search, screening and extraction of data whereas FL will review the work. JY, ZY and GX wrote the first manuscript draft and all authors gave input to the final draft of the protocol.

Funding This work was financially supported by the National Key Research and Development Program (N0.2019YFC1709701), the National Natural Science Foundation of China (No. 81590951, 81973962) and the Project of Science and Technology Department of Sichuan Province (No. 20ZDYF1199, 2019YFS0081).

Competing interests None declared.

Patient and public involvement Patients and/or the public were not involved in the design, or conduct, or reporting, or dissemination plans of this research.

Patient consent for publication Not required.

Provenance and peer review Not commissioned; externally peer-reviewed.

Open access This is an open access article distributed in accordance with the Creative Commons Attribution Non Commercial (CC BY-NC 4.0) license, which permits others to distribute, remix, adapt, build upon this work non-commercially, and license their derivative works on different terms, provided the original work is properly cited, appropriate credit is given, any changes made indicated, and the use is non-commercial. See: http://creativecommons.org/licenses/by-nc/4.0/.

\section{ORCID iDs}

Jiao Yang http://orcid.org/0000-0002-0323-2697

Guixing Xu http://orcid.org/0000-0002-8040-3656

Zihan Yin http://orcid.org/0000-0002-1543-0551

Ying Cheng http://orcid.org/0000-0003-3377-171X

Jiao Chen http://orcid.org/0000-0003-0830-6242

Fan-rong Liang http://orcid.org/0000-0001-8518-9268

\section{REFERENCES}

1 Sanford NN, Sher DJ, Butler SS, et al. Prevalence of chronic pain among cancer survivors in the United States, 2010-2017. Cancer 2019;125:4310-8.

2 Treede R-D, Rief W, Barke A, et al. Chronic pain as a symptom or a disease: the IASP classification of chronic pain for the International classification of diseases (ICD-11). Pain 2019;160:19-27.

3 Brown MR, Ramirez JD, Farquhar-Smith P. Pain in cancer survivors. Br J Pain 2014;8:139-53.

4 van den Beuken-van Everdingen MHJ, de Rijke JM, Kessels AG, et al. Prevalence of pain in patients with cancer: a systematic review of the past 40 years. Ann Oncol 2007;18:1437-49.

5 Burton AW, Fanciullo GJ, Beasley RD, et al. Chronic pain in the cancer survivor: a new frontier. Pain Med 2007;8:189-98.

6 Deng G, Cassileth BR. Integrative oncology: complementary therapies for pain, anxiety, and mood disturbance. CA Cancer J Clin 2005;55:109-16.

7 Hamood R, Hamood H, Merhasin I, et al. Chronic pain and other symptoms among breast cancer survivors: prevalence, predictors, and effects on quality of life. Breast Cancer Res Treat 2018;167:157-69.

8 Wiffen PJ, Wee B, Derry S, et al. Opioids for cancer pain - an overview of Cochrane reviews. Cochrane Database Syst Rev 2017;7:CD012592.

9 Giacalone A, Lleshi A, Zanet E, et al. Symptom burden in cancer survivors 1 year after diagnosis: a report from the American cancer Society's studies of cancer survivors. Cancer 2012;118:1955-6.

10 Fortner BV, Okon TA, Portenoy RK. A survey of pain-related hospitalizations, emergency department visits, and physician office visits reported by cancer patients with and without history of breakthrough pain. J Pain 2002;3:38-44.

11 Fallon M, Giusti R, Aielli F, et al. Management of cancer pain in adult patients: ESMO clinical practice guidelines. Ann Oncol 2018;29:iv166-91.

12 Swarm RA, Abernethy AP, Anghelescu DL, et al. NCCN clinical practice guidelines in Oncology:Adult Cancer Pain(Version3.2019). JNCCN 2019;11:992-1034. 
13 Benyamin R, Trescot AM, Datta S, et al. Opioid complications and side effects. Pain Physician 2008;11:S105-20.

14 American College of Chest Physicians. New ACCP cancer care guidelines include CAM. J Altern Complement Med 2007:13:1049.

15 Richardson MA, Sanders T, Palmer JL, et al. Complementary/ alternative medicine use in a comprehensive cancer center and the implications for oncology. J Clin Oncol 2000;18:2505-14.

16 MacPherson $\mathrm{H}$, Vertosick EA, Foster NE, et al. The persistence of the effects of acupuncture after a course of treatment: a meta-analysis of patients with chronic pain. Pain 2017;158:784.

17 Zhao L, Chen J, Li Y, et al. The long-term effect of acupuncture for migraine prophylaxis: a randomized clinical trial. JAMA Intern Med 2017;177:508-15.

18 Zhao L, Li D, Zheng $\mathrm{H}$, et al. Acupuncture as adjunctive therapy for chronic stable angina: a randomized clinical trial. JAMA Intern Med 2019;179:1388-97.

19 Hershman DL, Unger JM, Greenlee H, et al. Effect of acupuncture vs sham acupuncture or Waitlist control on joint pain related to aromatase inhibitors among women with early-stage breast cancer: a randomized clinical trial. JAMA 2018;320:167-76.

$20 \mathrm{He}$ Y, Guo X, May BH, et al. Clinical evidence for association of acupuncture and Acupressure with improved cancer pain: a systematic review and meta-analysis. JAMA Oncol 2019;6:271-8.

21 Peng H, Peng H-D, Xu L, et al. [Efficacy of acupuncture in treatment of cancer pain: a systematic review]. Zhong Xi Yi Jie He Xue Bao 2010;8:501-9.

22 Paley CA, Johnson MI, Tashani OA, et al. Acupuncture for cancer pain in adults. Cochrane Database Syst Rev 2015;2015:CD007753.

23 Choi T-Y, Lee MS, Kim T-H, et al. Acupuncture for the treatment of cancer pain: a systematic review of randomised clinical trials. Support Care Cancer 2012;20:1147-58.

24 Ruela LdeO, lunes DH, Nogueira DA, et al. Effectiveness of auricular acupuncture in the treatment of cancer pain: randomized clinical trial. Rev Esc Enferm USP 2018;52:e03402.

25 Chaimani A, Higgins JPT, Mavridis D, et al. Graphical tools for network meta-analysis in STATA. PLoS One 2013;8:e76654.

26 Salanti G, Del Giovane C, Chaimani A, et al. Evaluating the quality of evidence from a network meta-analysis. PLoS One 2014;9:e99682.

27 Moher D, Shamseer L, Clarke M, et al. Preferred reporting items for systematic review and meta-analysis protocols (PRISMA-P) 2015 statement. Syst Rev 2015;4:1.

28 Hutton B, Salanti G, Caldwell DM, et al. The PRISMA extension statement for reporting of systematic reviews incorporating network meta-analyses of health care interventions: checklist and explanations. Ann Intern Med 2015;162:777-84.

29 Lu W, Du P, Yang C, et al. The Effect of Computed TomographyGuided ${ }^{125}$ I Radioactive Particle Implantation in Treating Cancer and Its Pain. Cancer Biother Radiopharm 2018;33:176-81.
30 Zhang J, Zhang H, Luo Y. Association Between Activation of the Programmed Cell Death-1 (PD-1)/Programmed Death-Ligand 1 (PD-L1) Pathway and Pain in Patients with Cancer. Med Sci Monit 2019;25:1275-82.

31 Ham O-K, Chee W, Im E-O. The influence of social structure on cancer pain and quality of life. West J Nurs Res 2017;39:1547-66.

32 Edirisinghe NP, Makuloluwa TR, Amarasekara TD, et al. Psychometric properties of Sinhala version of short-form McGill pain Questionnaire-2 (SF MPQ-2-Sin) among patients with cancer pain in Sri Lanka. Pain Res Manag 2019;2019:1-6.

33 Reis-Pina P, Lawlor PG, Barbosa A. Adequacy of cancer-related pain management and predictors of undertreatment at referral to a pain clinic. J Pain Res 2017;10:2097-107.

34 Johnson JR, Burnell-Nugent M, Lossignol D, et al. Multicenter, double-blind, randomized, placebo-controlled, parallel-group study of the efficacy, safety, and tolerability of THC:CBD extract and $\mathrm{THC}$ extract in patients with intractable cancer-related pain. J Pain Symptom Manage 2010;39:167-79.

35 Huang Z, Su X, Diao Y, et al. Clinical consumption of opioid analgesics in China: a retrospective analysis of the National and regional data 2006-2016. J Pain Symptom Manage 2020;59:829-35.

36 Higgins JPT, Altman DG, Gøtzsche PC, et al. The Cochrane collaboration's tool for assessing risk of bias in randomised trials. BMJ 2011;343:d5928.

37 Guyatt GH, Oxman AD, Schünemann HJ, et al. GRADE guidelines: a new series of articles in the Journal of clinical epidemiology. J Clin Epidemiol 2011;64:380-2.

38 Cipriani A, Higgins JPT, Geddes JR, et al. Conceptual and technical challenges in network meta-analysis. Ann Intern Med 2013;159:130-7.

39 Portenoy RK, Ahmed E. Cancer pain syndromes. Hematol Oncol Clin North Am 2018;32:371-86.

40 Caldwell DM, Ades AE, Higgins JPT. Simultaneous comparison of multiple treatments: combining direct and indirect evidence. BMJ 2005;331:897-900

41 Salanti G. Indirect and mixed-treatment comparison, network, or multiple-treatments meta-analysis: many names, many benefits, many concerns for the next generation evidence synthesis tool. Res Synth Methods 2012;3:80-97.

42 Haidich AB. Meta-analysis in medical research. Hippokratian 2010;14:29-37.

43 Luce BR, Claxton K. Redefining the analytical approach to pharmacoeconomics. Health Econ 1999;8:187-9.

44 Spiegelhalter DJ, Abrams KR, Myles JP. Bayesian approaches to clinical trials and health-care evaluations. Vol. 13. . John Wiley \& Sons, 2004: 22. 80-5.

45 Schünemann HJ, Schünemann AHJ, Oxman AD, et al. Grading quality of evidence and strength of recommendations for diagnostic tests and strategies. BMJ 2008;336:1106-10. 